\title{
Design of a 2.5GHz Beam Scanning Antenna Array Controlled by Voltage Based on CRLH TL
}

\author{
Rui-kun Wu, Guo Tai Chen \\ Department of Electric and Information Engineering, Fuqing Branch of Fujian Normal University, Fujian 350300, China \\ e-mail: dzxi@china.com.cn
}

\begin{abstract}
An antenna array composed with power divider and small antenna at the operating frequency of $2.5 \mathrm{GHz}$ is designed and investigated, and the main radiation beam of the antenna array can be controlled by bias voltage. The measured results show that the main radiation beam of the antenna array has good directivity when the bias voltage of the varactor in the antenna array is changed from $8 \mathrm{~V}$ to $2 \mathrm{~V}$, and its scanning range is up to 96 degrees, which verifies that the antenna array radiation from backward to forward can be reached by controlling the bias voltage. Also, the power divider and small antenna are designed and analyzed respectively. The power divider and small antenna are designed on the basis of composite right/left-handed transmission line (CRLH TL).
\end{abstract}

Keywords-composite right/left-handed transmission line; power divider; antenna array

\section{INTRODUCTION}

Metamaterials was presented in theory and has been researched widely, since it has series of amazing electromagnetic properties, such as negative permittivity, negative permeability, negative phase velocity and positive group velocity. In the late 1990 s, an artificial medium having negative permittivity or negative permeability in the microwave frequencies was designed with metal thin-wire (TW) and metal split-ring resonator (SRR) was proposed in [1]. In [2], Smith et al first proposed artificial medium with negative permittivity and negative permeability simultaneously. This medium is combined TW and SRR structures in [1] into a composite structure. In 2002, Caloz et al proposed a left-handed matamaterials based on the transmission line method [3], where the left-handed matamaterials was constructed by the unit that consists of a series capacitor and a parallel inductor. In [4, 5], Caloz and Itoh applied left-handed transmission line theory to fabricate an left-handed microstrip transmission line with interdigital capacitors and shorted stub inductors, and verified it with the application of microwave devices. In antenna, Lei et al [6] proposed a left-handed transmission line leaky-wave scanning antenna which was constituted by interdigital capacitors and stub inductors shorted to the ground plane, and the interdigital capacitors and the stub inductors were engraved in a PCB board. The results in [6] showed that this antenna have radiation forward at higher frequencies and radiation backward at lower frequencies, and scanning radiation by changing the frequencies. In recent years, the authors have worked on the radiation characteristics of the composite right/left-handed transmission line (CRLH TL) leaky-wave antenna, and verified the radiation characteristics by the experiment [7].

In this paper, an antenna array constituted with power divider and small antenna is designed and measured. The operating frequency considered is $2.5 \mathrm{GHz}$ and the main radiation beam of the antenna array can be controlled by bias voltage. Based on composite right/left-handed transmission line theory described in Section II, a three-port power divider and small antenna is designed and the corresponding performances are analyzed by simulation or experimental measuring in Section III. And then the model and measured performances of the antenna array are presented in Section IV. Finally, the conclusion is drawn in Section V.

\section{COMPOSITE RIGHT/LEFT-HANDED TRANSIMISSION} LINE THEORY

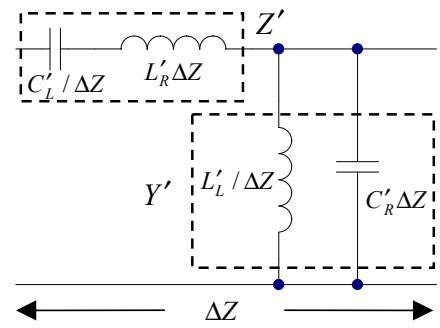

Figure 1. Equivalent circuit model for ideal CRLH TL

Usually, the equivalent circuit of an ideal loss-less CRLH TL [8] is shown in Figure 1, where $\triangle z$ is the length of an CRLH TL unit-cell. A unit-cell is consisted of a series LH capacitance $C_{L}^{\prime}$, a series $R H$ inductance $L_{R}^{\prime}$, a shunt $L H$ inductance $L_{L}^{\prime}$, and a shunt RH capacitance $C_{R}^{\prime}$. For a lossless transmission line, the per-unit-length impedance and admittance can be expressed respectively as follows.

$$
\begin{aligned}
& Z^{\prime}=\left(j \omega L_{R}^{\prime}+\frac{1}{j \omega C_{L}^{\prime}}\right) \\
& Y^{\prime}=\left(j \omega C_{R}^{\prime}+\frac{1}{j \omega L_{L}^{\prime}}\right)
\end{aligned}
$$

Then, the propagation constant is

$$
\begin{aligned}
\gamma & =\boldsymbol{j} \beta=\sqrt{\mathbf{Z}^{\prime} \boldsymbol{Y}^{\prime}} \\
& =\boldsymbol{j} \sqrt{\left(\omega \mathbf{L}_{R}^{\prime}-\frac{1}{\omega \boldsymbol{C}_{L}^{\prime}}\right)\left(\omega \boldsymbol{C}_{R}^{\prime}-\frac{1}{\omega \mathbf{L}_{L}^{\prime}}\right)}
\end{aligned}
$$

With $\beta_{L}=-1 /\left(\omega \sqrt{L_{L}^{\prime} C_{L}^{\prime}}\right)$ and $\beta_{R}=\omega \sqrt{L_{R}^{\prime} C_{R}^{\prime}}$, the propagation constant $\beta$ can be written as 


$$
\beta=\beta_{L}+\beta_{R}=\omega \sqrt{L_{R}^{\prime} C_{R}^{\prime}}-\frac{1}{\omega \sqrt{L_{L}^{\prime} C_{L}^{\prime}}}
$$

Figure 2 shows the frequency characteristic of the propagation constant $\beta$. It can be found that the propagation constant $\beta$ of CRLH TL changes from left region $(\beta<0)$ to right region $(\beta>0)$, and the zero is called balanced point for $\beta$, where

$$
\begin{aligned}
& \omega_{0}=\frac{1}{\sqrt{L_{R}^{\prime} C_{L}^{\prime}}}=\frac{1}{\sqrt{L_{L}^{\prime} C_{R}^{\prime}}} \\
& \text { the balance } \\
& \text { point, }
\end{aligned}
$$

At the balance point, i.e., propagation constants $\beta=\beta_{L}+\beta_{R}=0$, the wavelength of the electromagnetic wave is infinite, and electromagnetic wave do not radiate, which is an important feature of CRLH TL and can be used to design power divider.

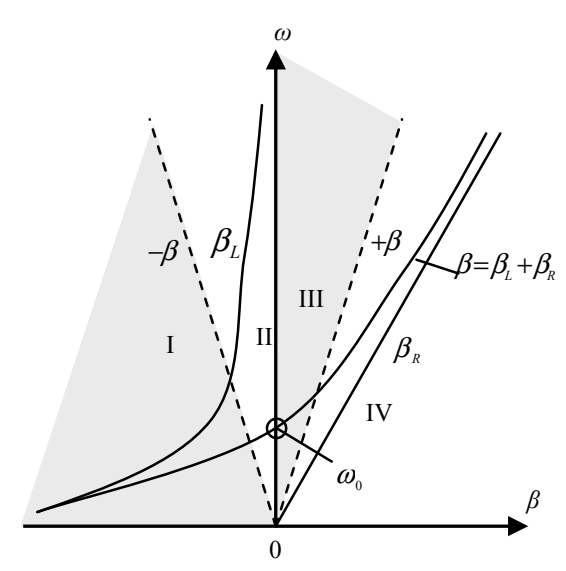

Figure 2. Dispersion curve of the CRLH TL.

The dispersion curve of CRLH TL can be divided to four areas which are labeled with I, II, III, and IV in Figure 2, respectively. Region I and region IV are left-handed guided wave model and right-handed guided wave model respectively, and region II and III are the radiation modes of left-handed and right-handed respectively. As a microstrip leaky-wave antenna, CRLH TL can only work in the region II and region III. In these two regions, because the value of $\beta$ changed from negative, zero to positive, a microstrip leakywave antenna based on CRLH TL working in these two regions can realize backward radiation, endfire radiation and forward radiation [9].

The radiation angle of the microstrip leaky-wave antenna can be written

$$
\theta=\arccos \left(\left(\beta_{0}(\omega)+2 \boldsymbol{n} \pi\right) / \boldsymbol{k}_{0}\right)
$$

Where $\beta_{0}(\omega)$ represents the phase constant of the fundamental model, $\boldsymbol{n}(\boldsymbol{n}=0, \pm 1, \pm 2, \cdots)$ is the number of the space harmonics, and $\boldsymbol{k}_{0}$ for the wave vector of the free space.

\section{Design for Power Divider AND SMAll ANTENNA}

\section{A. Design for Power Divider}

Power divider is often restricted by the location of the antenna unit in the design of antenna array. However, the shortcomings of traditional power divider can be overcome by the series power divider based on CRLH TL, which can support an infinite wavelength at resonant frequency. At the resonant frequency, the signal amplitude and phase of CRLH TL are the same at any position, which weakens the requirements of the physical length of power divider and the location of power feed, and makes power divider have different number of feed ports. The model of an $N$-port power divider is shown in Figure 3, which $\boldsymbol{d}_{\boldsymbol{i}}$ is the distance between of the output ports of power divider [10].

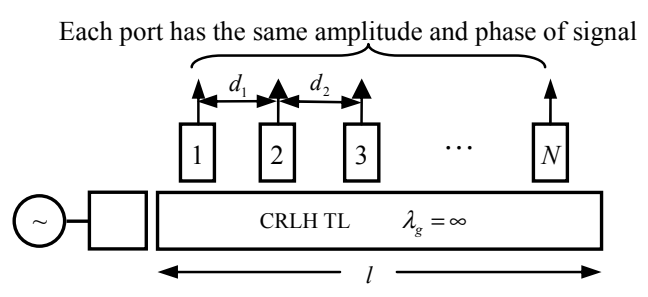

Figure 3. $N$-port power divider of the infinite wavelength.

The power divider designed with CRLH TL is practically connected with the unit-cell shown in Figure 1, as the terminal open or terminal short resonator, and the resonant frequency can be obtained by using Bloch-Floquet theory to solve the dispersion relation [11].

$$
\begin{aligned}
\beta_{n} \boldsymbol{d} & =\frac{n \pi \Delta \boldsymbol{z}}{\boldsymbol{l}}=\frac{\boldsymbol{n} \pi}{\boldsymbol{N}} \\
& =\cos ^{-1}\left\{1-\frac{1}{2}\left[\frac{\omega_{L}^{2}}{\omega_{n}^{2}}+\frac{\omega_{n}^{2}}{\omega_{R}^{2}}-\left(\frac{\omega_{L}^{2}}{\omega_{s e}^{2}}+\frac{\omega_{L}^{2}}{\omega_{s h}^{2}}\right)\right]\right\}
\end{aligned}
$$

Here $\omega_{L}=1 / \sqrt{L_{L}^{\prime} C_{L}^{\prime}}, \omega_{R}=1 / \sqrt{L_{R}^{\prime} C_{R}^{\prime}}, \omega_{s e}=1 / \sqrt{L_{R}^{\prime} C_{L}^{\prime}}$, $\omega_{s h}=1 / \sqrt{L_{L}^{\prime} C_{R}^{\prime}}, \triangle z$ is the unit length of the CRLH TL, $N$ is the number of units of the CRLH TL resonator, and $n$ for the resonant mode.

For the zero order status $(\beta \rightarrow 0)$ of the terminal open resonator with CRLH TL, the parallel resonant frequency is $\omega=\omega_{s h}$. When the resonator works at the fundamental mode, the transmission line presents the state of standing wave, and all points along the transmission line have the same amplitude and phase of signal, this feature is one requirement of the antenna array feed network.

A series power divider simulation model with three output ports is shown in Figure 4, where I-port is the signal input port, the right is the terminal open port. The ports located above are the output port, there are port 1 , port 2 and port 3 , respectively. The dielectric constant of microstrip transmission line is 2.0 , and the thickness is $2 \mathrm{~mm}$. In the simulation, the unit length, total length and microstrip transmission line width of the power divider are set as 
$12.47 \mathrm{~mm}, \quad 143.90 \mathrm{~mm}$ and $6.20 \mathrm{~mm}$, respectively, by simulation optimization.

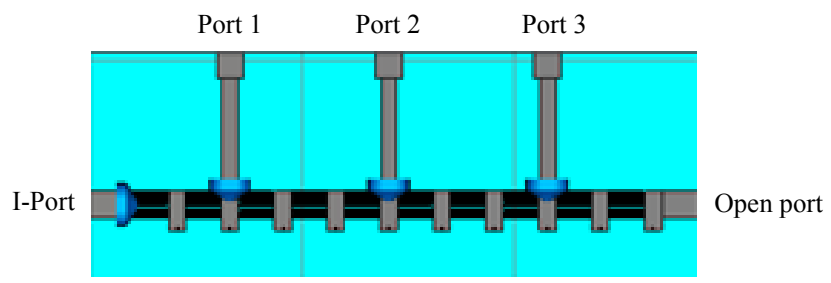

Figure 4. Power divider of the CRLH TL.

The simulation results of amplitude-frequency characteristics and phase-frequency characteristics of the power divider are shown in Figure 5. As shown in the figure, at the frequency of $f=2.5 \mathrm{GHz}$, the signal amplitude are $12.76 \mathrm{~dB},-9.78 \mathrm{~dB}$ and $-12.23 \mathrm{~dB}$ from port 1 to port 3 respectively, and the corresponding phases are -68.05 , 76.13 and -80.96 degrees respectively. Compared with the results at other frequencies in the figure, the signal amplitude and phase of each output port at $2.5 \mathrm{GHz}$ are very close and can be approximately equal, which basically meets the requirements of the power divider.

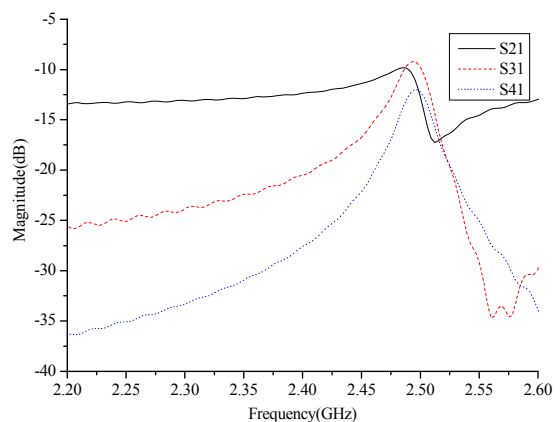

(a) Amplitude-frequency characteristic

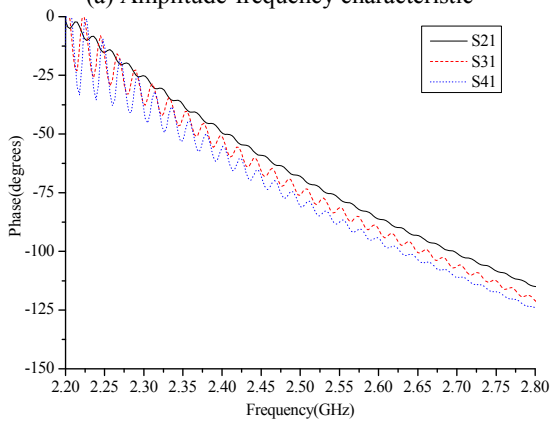

(b) Phase-frequency characteristic

Figure 5. Simulation results of the power divider.

\section{B. Design for Small Antenna}

Based on the theory of CRLH TL, we design a small antenna and engrave it on the PCB board with dielectric parameter 2.0 and thickness $2 \mathrm{~mm}$. The antenna is comprised with seven units, and the length and width are $106.5 \mathrm{~mm}$ and $35 \mathrm{~mm}$ respectively. Each unit with length $13.36 \mathrm{~mm}$ is carved with interdigital capacitor and stub inductor, and loaded with three varactors. A matching resistor of $50 \Omega$ is soldered on the antenna terminal.

The S-parameters of the small antenna are measured by vector network analyzer and presented in Figure 6. It can be found that S11 has a series of low reflection band near the work frequency of $2.5 \mathrm{GHz}$ when the bias voltage of the varactors is changed from $2 \mathrm{~V}$ to $8 \mathrm{~V}$. This corresponds to the right handed passband of the S21, where the capacitance value of the varactor decreases with the bias voltage increases, and S11 curve clusters move along the increasing direction of frequency.

The results of S21 show that S21 changes from the righthanded passband to the gap and then to the left-handed passband with the bias voltage increases. Such change means that the main radiation direction of the CRLH TL antenna is changing, namely the low biasing voltage corresponds to the forward radiation of the antenna and the high biasing voltage corresponds to the backward radiation.

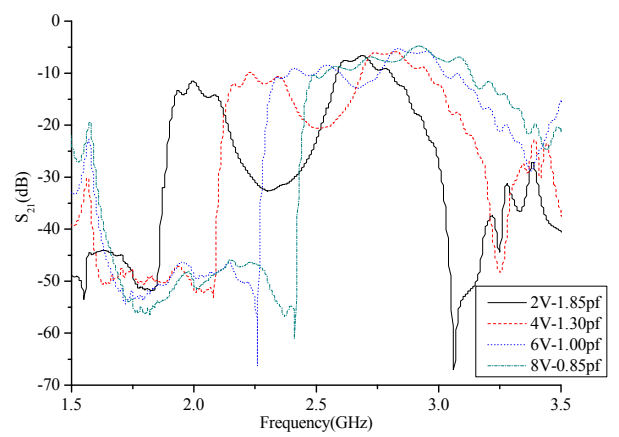

(a) $\mathrm{S} 21$

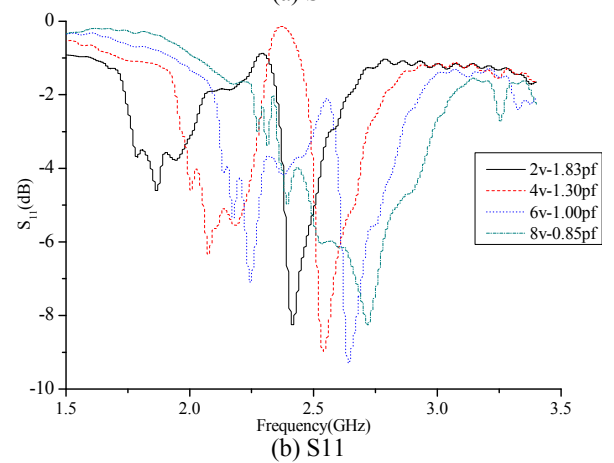

Figure 6. Measurements of S-parameter of the small antenna.

\section{ANTENNA ARRAY}

In this section, we constitute an antenna array with the power divider and three small antennas designed in the last section. Figure 7 shows the model of antenna array, where the length and width are $190 \mathrm{~mm}$ and $150 \mathrm{~mm}$ respectively. 


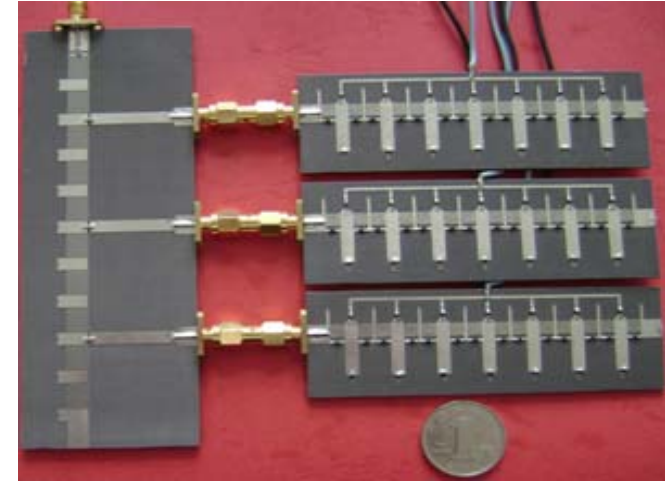

Figure 7. Antenna array.

The horizontal polarization patterns of the antenna array are measured in an anechoic chamber, and the results with the different bias voltage are presented in Figure 8.

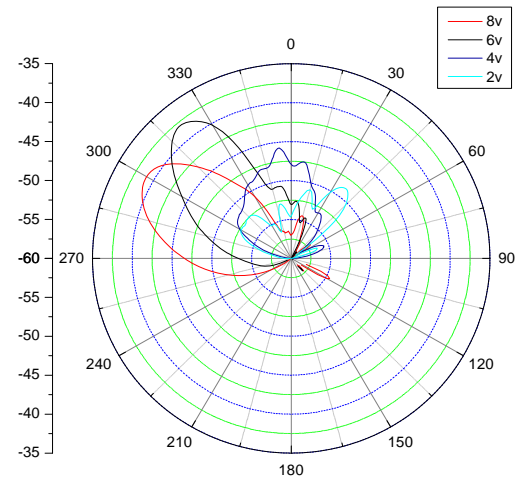

Figure 8. Antenna horizontal polarization pattern.

The results show that the radiation beam of the CRLH TL antenna array has a good directivity at the operating frequency of $2.5 \mathrm{GHz}$ from the figure. When the varactor bias voltage is $8 \mathrm{~V}$, the main beam radiation angle and the gain of the antenna array are -60 degrees and $-39.1 \mathrm{~dB}$ respectively. The main beam radiation angle and the gain are -42 degrees and $-38.1 \mathrm{~dB}$ when the bias voltage is $6 \mathrm{~V}$, and -7 degrees and $-38.1 \mathrm{~dB}$ for $4 \mathrm{~V}$. When the bias voltage is changed to $2 \mathrm{~V}$, the radiation angle and the gain of the antenna array are 36 degrees and $-48.7 \mathrm{~dB}$ respectively.

These results verify that the presented antenna array has the good performance of the beam scanning. When the bias voltage is reduced from $8 \mathrm{~V}$ to $2 \mathrm{~V}$, the beam scanning radiation angle of the antenna array can reach 96 degrees, and the radiation beam of the antenna array changes from the backward to the forward.

\section{CONCLUSION}

An antenna array comprised with CRLH TL power divider and small antennas have been presented and the performances also have been measured and analyzed. The results show that the main radiation beam has good scanning performance. The scanning angle of the antenna array main radiation beam is about 96 degrees by changing the bias voltage from $8 \mathrm{~V}$ to $2 \mathrm{~V}$ at $2.5 \mathrm{GHz}$. The antenna array has the advantages of simple structure, small size, scan features, and can be applied to wireless local networks and short distance wireless. The CRLH TL power divider and small antenna are also independently investigated in this paper.

\section{ACKNOWLEDGMENT}

This work is supported by the Fujian Province Natural Science Foundation funded projects (2010J05127) and the Fujian Province Department of Education funded project (JK2011064), it also is supported by the Fuqing Branch of Fujian Normal University research projects (KY2011008).

\section{REFERENCES}

[1] Cui Wanzhao, Ma Wei, Qiu Lede, et al. Electromagnetic Metamaterials and its applications [M]. Beijing: National Defense Industry Press, 2008:4-16.

[2] D. R. Smith, D. C. Vier, Willie Padilla, et al. Loop-wire medium for investigating plasmons at microwave frequencies $[\mathrm{J}]$. Applied Physics Letters, 1999, 75(10): 1425-1427.

[3] C. Caloz, H. Okabe, T. Iwai, et al. Transmission line approach of lefthanded (LH) materials [C]. USNC/URSI National Radio Science Meeting, San Antonio, TX, 2002, 39:39-41.

[4] C. Caloz, T. Itoh. Application of the transmission line theory of lefthanded (LH) materials to the realization of a microstrip "LH line" [C]. IEEE Ante. Prop. Soci. Int. Symp. 2002, vol.2: 412-415.

[5] C. Caloz, T. Itoh, et al. Transmission line approach of left-handed (LH) materials and microstrip implementation of an artificial LH transmission line $[\mathrm{J}]$. IEEE TRANSACTIONS ON ANTENNAS AND PROPAGATION, 2004, vol. 52:1159-1166.

[6] L. Lei, C. Caloz, T. Itoh. Dominant mode leaky wave antenna with backfire-to-endfire scanning capability [J], Electron. Lett., 2002, vol. 38: 1414-1416.

[7] Wu Ruikun. Investigation of Radiation Characteristics for Metamaterial Microstrip Leaky-Wave Antennas [J]. Journal of Microwaves, 2011, 27(1):44-47.

[8] C. Caloz, A. Sanada, and T. Itoh. A novel composite right/left-handed coupled-line directional coupler with arbitrary coupling level and broad bandwidth [J]. IEEE Trans. Microwave Theory Tech., 2004, vol.52:980-992.

[9] Zhang Yewen, $\mathrm{Li}$ Guiquan, $\mathrm{He} \mathrm{Li}$, et al. One-Dimensional Metamaterial-Based Microstrip Leaky-Wave Antenna with Backfireto-Endfire Scanning Capability [J]. Journal of Tongji University(Natural Science), 2007, vol.35:811- 814.

[10] A. Lai, K. M. K. H. Leong, T. Itoh. A novel n-port series divider using infinite wavelength phenomena [C]. IEEE MTT-S International Microwave Symposium Digest, 2005:1001- 1004.

[11] A. Sanda, C. Caloz, T. Itoh. Novel zeroth-order resonance in composite right/left-handed transmission Line Resonators [C]. AsiaPacific Microwave Conference, 2005, vol.3:1588- 1592. 